\title{
Recent Insights into the Role of Gut Microbiota in Diabetic Retinopathy
}

\author{
Jinghua Jiao $\mathbb{D}^{1, *}$ \\ Honghua $\mathrm{Yu}^{2, *}$ \\ Litong $\mathrm{Yao}^{3, *}$ \\ Lihua $\mathrm{Li}^{4}$ \\ Xiaohong Yang ${ }^{2}$ \\ Lei Liu (1D ${ }^{2}$ \\ 'Department of Anesthesiology, Central \\ Hospital, Shenyang Medical College, \\ Shenyang, Liaoning, I I0024, People's \\ Republic of China; ${ }^{2}$ Guangdong Eye \\ Institute, Department of Ophthalmology, \\ Guangdong Provincial People's Hospital, \\ Guangdong Academy of Medical Sciences, \\ Guangzhou, Guangdong, 5I0I20, People's \\ Republic of China; ${ }^{3}$ Department of Breast \\ Surgery, The First Affiliated Hospital of \\ China Medical University, Shenyang, \\ Liaoning, I I000I, People's Republic of \\ China; ${ }^{4}$ Department of Ophthalmology, \\ Affiliated Hospital of Weifang Medical \\ University, Weifang, Shandong, 261031, \\ People's Republic of China
}

*These authors contributed equally to this work
Correspondence: Xiaohong Yang; Lei Liu Email syyangxh@scut.edu.cn;

liuleijiao@163.com

\begin{abstract}
The microbiome has become a hot issue in recent years. The composition, modification, alteration, and disturbance of gut microbiota were found to influence important physiological processes, including energy metabolism and microenvironmental homeostasis, and lead to various diseases, including obesity, type 2 diabetes mellitus and chronic kidney disease. Diabetic retinopathy (DR) is a major microvascular complication of diabetes mellitus and one of the leading causes of blindness and vision impairment. The underlying mechanisms in DR pathogenesis remain limited. Recently, important insights have been made regarding possible connections between gut microbiome dysbiosis and ocular disease including DR, uveitis, glaucoma, and age-related macular degeneration, and the concept of a "microbiota-gut-retina axis" has been put forward. Hence, we reviewed current understanding of the relationship between DR and gut microbiota. We summarized potential pathophysiological mechanisms that contribute to the role of the gut microbiota on DR, including hyperglycemia, anti-diabetes drugs, microbial metabolites, and inflammatory properties. We aimed to find novel effective therapeutic options to prevent the onset and development of DR.
\end{abstract}

Keywords: retinopathy, bacteria, microbiota, microbiota-gut-retina axis, mechanism

\section{Introduction}

Diabetic retinopathy (DR) is one of the most common complications of diabetes mellitus (DM), ${ }^{1}$ and it is estimated that there will be nearly 191 million people with the disease by $2030 .^{2,3}$ In addition, DR has become one of the leading causes of blindness and vision impairment in the working age population. ${ }^{4-6}$ A recent report indicated that cases of blindness due to DR may cost up to $\$ 500$ million annually in the United States. ${ }^{7}$ Based on current epidemiologic investigations and clinical studies, the onset and progression of DR is complicated and has been associated with various factors, including duration of disease, glycemia and blood pressure control. ${ }^{8,9}$ However, the underlying molecular mechanisms in DR pathogenesis remain limited and unclear. The lack of effective therapeutic options for the prevention of DR necessitates further investigation of pathological mechanisms and effective approaches that would provide new therapeutic strategies against DR.

The microbiome has become a hot issue of concern for research in recent years. Previous evidence has demonstrated that the composition, modification and disturbance of gut microbiota affect important physiological processes, including energy metabolism and microenvironmental homeostasis. ${ }^{10,11}$ The gut dysbiosis held great significance in susceptibility to and development of various diseases in vivo in rodent and human studies, including obesity, type 2 diabetes mellitus 
(T2DM), chronic kidney disease (CKD) $)^{12,13}$ and rheumatoid arthritis (RA). ${ }^{14}$ Subsequent studies reported that gut microbiota could lead to chronic inflammation and imbalance of immune system referring to small-molecule constituents such as short-chain fatty acids (SCFA) and secondary bile acids and trim-ethylamine. ${ }^{15}$ The relationship between gut microbiota and ocular abnormalities including uveitis, glaucoma, and age-related macular degeneration (AMD) has been increasingly revealed, and the concept of a "microbiota-gut-retina axis" has been put forward. ${ }^{16,17}$ However, little has been understood regarding the molecular mechanisms of intestinal dysbiosis and how they induce retinopathy in patients with DM. Recently, data indicate that changes in the gut microbiome act as a key internal environmental modulator which favorably affects the pathogenetic processes underlying the micro-complications of DM such as DR. ${ }^{18}$

In this review, we propose to highlight the several potential pathophysiological mechanisms that contribute to the influence of the gut microbiota on DR, including hyperglycemia, anti-diabetes drugs, microbial metabolites and inflammatory properties. To achieve this, we searched electronic databases including PubMed, Web of Science, the Cochrane Central Register of Controlled Trials, EMBASE, OVID, China National Knowledge Infrastructure (CNKI), Wanfang and Weipu, using the following search terms: "diabetic retinopathy", "retinopathy", "retina", "gut microbiota", "gut flora", "gastrointestinal bacteria" and "gastrointestinal flora". We included English or Chinese language articles and other content including clinical and experimental studies regarding DR up to January 2021. We took into consideration articles that contributed to the discussion of gut microbiota which played a role in the pathophysiology of DR.

\section{Main Mechanisms of DR Hyperglycemia}

As already widely known, DR is related to several dominant risk factors such as hyperglycemia, hypertension, dyslipidemia and obesity, and hyperglycemia has a major impact on the modulation of metabolism. ${ }^{4,19}$ The Diabetes Control and Complications Trial (DCCT) showed that intensive therapy reduced the risk of the development and progression of diabetic retinopathy. ${ }^{20}$ In addition, the United Kingdom Prospective Diabetes Study (UKPDS) confirmed a similar significant relationship among the degree of hyperglycemia and the incidence of DR in patients with T2DM. ${ }^{21}$
Generally, the mechanism regarding hyperglycemia induced micro-angiopathy included oxidative stress and glucosemediated endothelium dysfunction. In line with that, hyperglycemia induced a significant increase in reactive oxygen species (ROS) production through glucose autoxidation or the activity of cytosolic nicotinamide adenine dinucleotide phosphate (NADPH) oxidase, or regulating mitochondrial respiratory chain. ${ }^{22}$ Previous studies indicated that ROS could lead to the impairment of the antioxidant defense system and influence retinal metabolic abnormalities. Furthermore, endothelium cell dysfunction can be caused by the production of toxic metabolites which interfere with glucose-mediated endothelium damage. In view of current knowledge, excess glucose inside the cells is converted to sorbitol by aldose reductase (AR), and sorbitol is further oxidized to fructose by sorbitol dehydrogenase (SDH). ${ }^{23}$ When aldose reductase oxidizes NADPH to $\mathrm{NADP}^{(+)}$, $\mathrm{SDH}$ uses $\mathrm{NAD}^{(+)}$to form nicotinamide adenine diphosphate hydride (NADH). This activation of the polyol pathway can reduce NADPH and $\mathrm{NAD}^{(+)}$levels, leading to the occurrence of redox in the body. ${ }^{24}$ In addition, hyperglycemia increases the thickness of the capillary basement membrane in the nerve fiber and outer plexiform layer, reduces the area of pericytes and increases the number of blood vessels, makes retinal capillary cells apoptotic, and decreases retinal dismutase as well as peroxidation catalase activity. ${ }^{25,26}$ Moreover, hyperglycemia-related retinal pigment epithelial cell apoptosis and inflammation play a vital role in the development of DR.

\section{Inflammation}

Inflammation is a key process in metazoan organisms due to its relevance for innate defense against infections and tissue damage. However, inflammation is also implicated in pathological processes such as DM. Notably, an aberrant immune response following exposure to non-pathogenic stimuli in diabetes involving chronically relapsing inflammatory conditions is related to the pathogenesis of the disease and its complications. In a pre-print work, Luo et $\mathrm{al}^{27}$ have shown that inflammation participates in several events involved in DR pathogenesis. Especially, chronic disorders characterized by inflammation are also involved in the development of micro-complications of $\mathrm{DM} .{ }^{28}$ Increased concentrations of pro-inflammatory cytokines were discovered in the serum, aqueous humor and vitreous of patients with DR compared with those without DR. During the inflammatory response, the increased expression of vascular endothelial growth factor (VEGF) 
production can promote retinal pigment epithelial (RPE) cell proliferation, and increase vascular permeability, leading to inner retinal and vitreous hemorrhage. ${ }^{29}$ VEGF pathways play significant roles in regulating the expression of intercellular adhesion molecule-1 (ICAM-1) in endothelial cells, and activating leukocytes to release some cytokines, which contributes to an increase in the expression of VEGF, and further aggravates the inflammatory response. ${ }^{30}$ At present, intraocular injection of antiVEGF drugs is widely applied in the treatment of retinal or choroidal neovascular disorders such as DR, retinopathy of prematurity, and AMD. ${ }^{31,32}$ A large number of experiments have shown that VEGF can be produced by retinal glial (Müller) cells. The inhibition of glial cells can reduce the expression of tumor necrosis factor (TNF) - $\alpha$, ICAM1 , etc. ${ }^{33,34}$ The inflammatory response also directly interacts with cell adhesion and a breakdown of the blood-retinal barrier (BRB). ${ }^{35}$ The expression of inflammatory protein is increased by the activation of pro-inflammatory transcription factors. In patients with proliferative $\mathrm{DR}$, the expression of nuclear factor (NF) $-\mathrm{kB}$ is significantly higher than that of non-DR individuals. ${ }^{36}$ Activation of NF- $\kappa \mathrm{B}$ eventually brings about the synthesis of a variety of cytokines, chemokines, acute-phase proteins and pro-inflammatory molecules, mediating the production of inflammatory responses.

Given the crucial role of inflammation in the regulation of immune responses in retinopathy and the maintenance of metabolic health, we believe that further studies are necessary to improve our knowledge concerning possible pathophysiological mechanisms in the context of DR.

\section{Evidence that Gut Microbiota May Be Associated with DR}

Nowadays, the gut microbiome has become a focus of much research, with both human studies and rodent models. Recent findings suggested that there is a significant association between gut dysbiosis and various disorders, such as hypertension, cardiovascular diseases (CVDs), diabetes and obesity. ${ }^{37-39}$ Larsen et al initially confirmed the relationship between gut microbiota (Firmucutes and Bacteroidetes) and diabetes. Diabetes is associated with lower relative abundance of Firmicutes, while the proportion of Bacteroidetes is higher. ${ }^{40}$ Subsequently, further investigations indicated that microbial dysbiosis was related to systematic biochemical metabolism, insulin resistance and $\mathrm{T} 2 \mathrm{DM}$, uncovering the function of the microbiome in diabetes. The increased understanding of gut microbiota potentially provides effective therapeutic approaches for prevention and management of diabetes. ${ }^{41}$ However, the role of microbial dysbiosis in pathogenesis of diabetic microvascular complications, such as DR or nephropathy, remains elusive. ${ }^{42}$ A potential relationship between microbiome and DR, even indirect, may exist. In fact, metabolic factors associated with chronic lowgrade inflammation and oxidative stress, that link altered intestinal microbiota composition and T2DM, are also influencing the onset and progression of DR. Generally, gut microbiota dysbiosis leads to continuous inflammation and immunosenescence, both of which may be associated with the pathogenesis of DR (Figure 1).

A previous study in a diabetic $\mathrm{db} / \mathrm{db}$ mice model of diabetes has provided evidence regarding effects of intermittent fasting (IF) on the development of DR, and the IF regimen could lead to a reduction of retinopathy, including acellular capillaries and immune infiltration. ${ }^{43}$ Interestingly, researchers found that IF increased levels of Firmicutes but decreased Bacteroidetes and Verrucomicrobia. Further, they demonstrated the positive effect of IF on improvement of the gut barrier, that is, that IF could improve gut structure in a db/ $\mathrm{db}$ mice, including increased gut mucin, goblet cell number, and villi length and reduced peptidoglycan. Notably, IF increased expression levels of Tauroursodeoxycholate (TUDCA; UR 906; Taurolite), which is an endoplasmic reticulum (ER) stress inhibitor as well as a secondary bile acid with neuroprotective properties. ${ }^{44}$ TUDCA modulated the glycolipid metabolism through farnesoid $\mathrm{X}$ receptor (FXR) and bile acid G-protein-coupled membrane receptor TGR5. Rizzo et al have indicated the functional characterization of INT-767, a potent and selective FXR receptor and TGR5 agonist, suggesting their potential application in liver and metabolic diseases treatment. ${ }^{45}$ Additionally, INT-767 is a safe and effective modulator of FXR and TGR5-dependent pathways, suggesting potential clinical applications in the treatment of metabolic disorders and their complications. ${ }^{46}$ Beli et al found that INT-767 could prevent DR in diabetic mice model prevent. ${ }^{43}$ Collectively, these preclinical findings indicate that IF intervention modified the gut microbiota toward species, and produced TUDCA, which promoted TGR5 activation in retinal neurons and subsequently prevented DR by TGR 5 activation in retinal neurons. Recently, Das et al demonstrated the alteration in the diversity and abundance of gut microbiota in patients with T2DM and DR. Gut microbiomes of DR patients were found to be discriminatory. Anti-inflammatory, probiotic, and possibly 


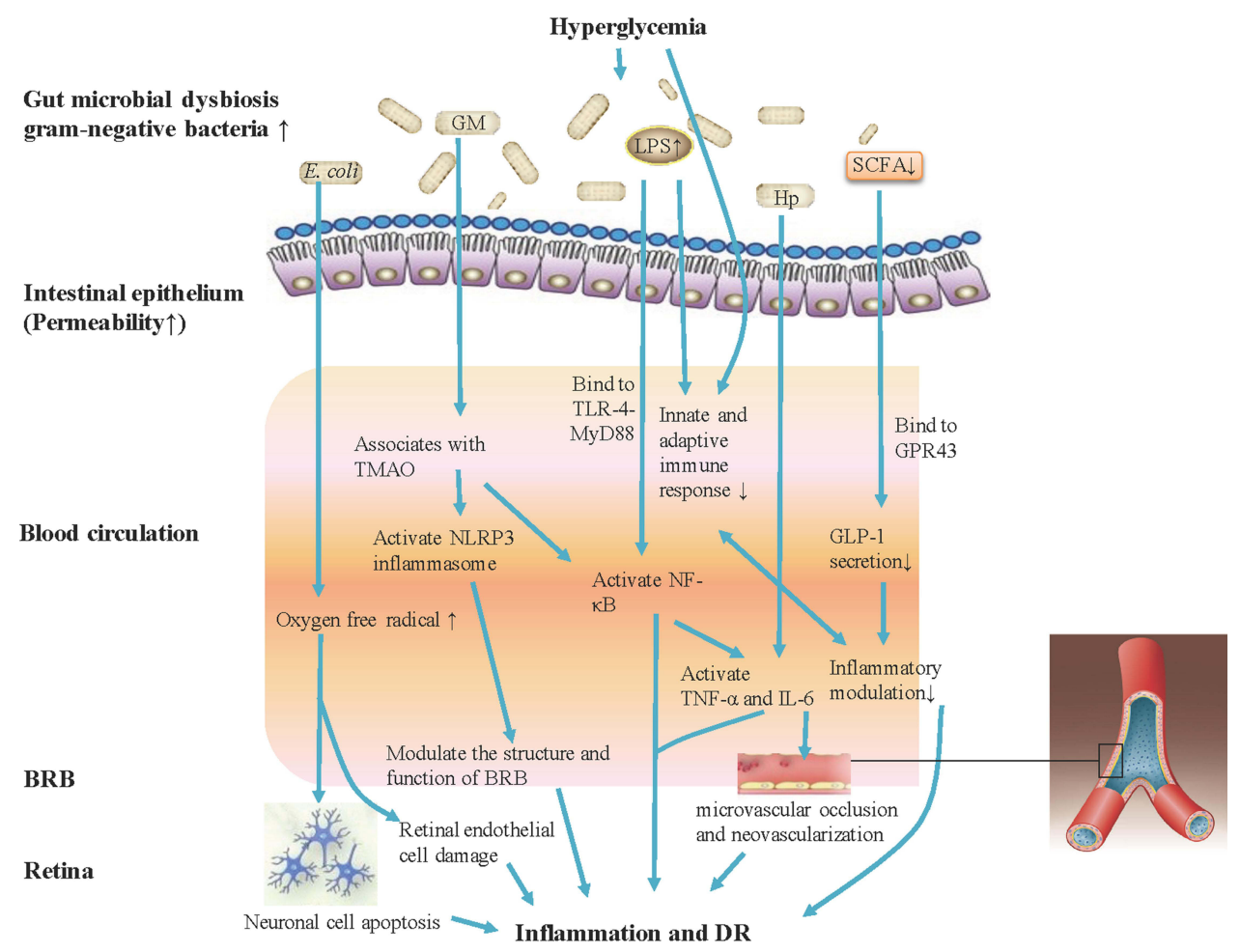

Figure I Gut microbiota modulates inflammation and immune mechanism of hyperglycemia-induced DR. Gut microbiota dysbiosis under hyperglycemia with type 2 diabetes (T2DM) causes bacterial translocation and gram-negative bacteria increase allowing accumulated production of LPS into blood circulation. Elevated LPS level activates NF- $K B$ through TLR-4-MyD88, increases the expression of IL-6 and TNF- $\alpha$, and also can deregulate the innate and adaptive immune response. Increased Hp can induce the expression of IL- 6 and TNF- $\alpha$, and IL- 6 can cause damage to vascular endothelial cells. The increasing abundance of $E$. coli in the microbiome of patients with T2DM will lead to the generation of oxygen free radicals, causing neuronal cell apoptosis and extensive damage to retinal endothelial cells, and promote the development of DR. Gut microbiota associates with the serum levels of TMAO, which promotes vascular inflammation by activating inflammasome, NLRP3, through reactive oxygen species (ROS) signaling pathway. Further, the NLRP3 inflammasome modulates the structure and function of BRB to induce damage of retina. Patients with T2DM commonly have a moderate degree of gut microbial dysbiosis, the abundance of some bacteria that produce butyrate reduction, and impact the levels of SCFA. SCFA production by colonic fermentation, which binds to the GPR43, regulates inflammation and GLP-I secretion, and further influences the development of DR.

Abbreviations: DR, diabetic retinopathy; LPS, lipopolysaccharide; TLR-4-MyD88, toll-like receptor 4/myeloid differentiation primary RB response 88; Hp, H. pylori; TMAO, trimethylamine-N-oxide; NLRP3, nucleotide-binding oligomerization domain-like receptor family pyrin domain-containing 3; BRB, blood-retinal barrier; SCFA, short-chain fatty acid; GPR43, G-protein-coupled receptor-43; GLP-I, glucogon-like-peptide-I.

pathogenic bacteria were reducsed in people with DR in comparison to healthy people and patients with T2DM. DR microbiomes performed a decrease in the pro-inflammatory organisms Sutterella, probiotic bacterium Lactobacillus and possibly pathogenic bacteria including Clostridium, Haemophilus, Erwinia, Desulfovibrio, Bulleida, Rothia, and Comamonas. Similarly, anti-inflammatory bacterium including Roseburia, Lachnospira, Blautia, Faecalibacterium, Bifidobacterium, and Ruminococcus were reduced in people with DR in comparison to healthy controls and patients with T2DM. ${ }^{47}$

\section{Hypothesis that Gut Microbiota Cause DR}

\section{Inflammation}

Interestingly, gut microbiota dysbiosis under hyperglycemia with type 2 diabetes causes bacterial translocation and intestinal endotoxin accumulation, resulting in intestinal inflammation. ${ }^{48}$ Insoluble LPS can be present in the gut and pass into the bloodstream. ${ }^{49}$ Lipopolysaccharide (LPS) was proven to be a major component of microbial translocation during chronic inflammation. ${ }^{50}$ LPS derived from gramnegative bacteria and elevated in the intestine of patients with T2DM was proven to cause metabolic endotoxemia, disrupt the arrangement of tight junction proteins, increase intestinal permeability and damage the intestinal epithelial barrier, thereby allowing intestinal bacterial metabolites to enter blood circulation. ${ }^{51-53}$ LPS-mediated activation of tolllike receptor 4 (TLR-4) complex could induce the myeloid differentiation primary-response protein (MyD88) and the tumor necrosis factor receptor-associated factor-6 (TRAF6 ), and subsequently lead to release of NF-kB/Rel family members. ${ }^{54}$ The disorder in intestinal flora composition and function and increasing levels of Helicobacter Pylori (H. pylori) can also induce the expression of IL-6 and 
TNF- $\alpha$. The secretion of these inflammatory cytokines can cause damage to vascular endothelial cells. ${ }^{55,56}$ The destruction of vascular endothelial cells induces disruption of vascular tone and hemodynamic changes, resulting in the activation of the coagulation and complementary systems, enhancing vascular permeability, and ultimately leading to microvascular occlusion and development of new blood vessels (retinal neovascularization), which exacerbates the occurrence of DR or diabetic macular edema (DME). ${ }^{57-59}$ Moreover, Huang et al recently found an increased level of gram-negative bacteria Bacteroidetes and Desulfobacterota phyla in participants with DR in comparison to those with DM. LPS derived from Bacteroidetes and Desulfobacterota could generate inflammatory injuries, trigger abnormal equilibrium of metabolism, and inhibit endotoxin tolerance, all of which are related to the pathogenesis of retinopathy. ${ }^{18,60}$ Influence of endotoxin-mediated retinal inflammation on phenotype of diabetic retinopathy. Additionally, Vagaja et al revealed that endotoxin-mediated retinal inflammation may have impact on phenotype of DR, that is, the systemic LPS exposure in Ins2 Akita mice could accelerate the damage of retinal capillary endothelium and attenuation of the retina. ${ }^{61}$ In addition, the increased abundance of Escherichia coli (E. coli) in the microbiome of patients with type 2 diabetes increases the level of uric acid, leading to the generation of oxygen free radicals, causing neuronal cell apoptosis and extensive damage to retinal endothelial cells, and also promotes the onset of DR. ${ }^{62}$ High glucose level could induce toll-like receptor (TLR-4) expression in retinal ganglion cells (RGCs), regulate its signaling pathways and increase the expression of inflammation cytokines such as TNF- $\alpha$, IL- 8 , and NF- $\kappa \mathrm{B}$, and thus promote glycosylation enzyme activity, activate microglia, increase vascular permeability and generate neuronal damage, which ultimately exacerbate retinopathy. ${ }^{63,64}$

Generally, gut microbial metabolism of choline leads to the formation of trimethylamine (TMA) and is the precursor of trimethylamine-N-Oxide (TMAO). In vivo, the serum levels of TMAO are associated with T2DM. ${ }^{65}$ Research suggested that individuals with an unhealthy body mass index (BMI) or abnormal glycemia were associated with higher serum levels of TMAO. TMAO promotes vascular inflammation by activating inflammasome, a nucleotidebinding oligomerization domain-like receptor family pyrin domain-containing 3 (NLRP3), through the ROS signaling pathway. Activation of NLPR3 inflammasome could be triggered by active oxygen species generation. ${ }^{66,67}$ Previous reports have revealed that the activation of
NLRP3 inflammasome is related to the pathogenesis of PDR. ${ }^{68-70}$ Compared with healthy controls, NLRP3 gene and protein expression were increased in peripheral blood mononuclear cells in patients with DR, and IL-1 $\beta$ and IL-18 levels were also elevated in peripheral blood mononuclear cells and vitreous fluid in patients with DR. ${ }^{71}$ TMAO can also promote the phosphorylation of NF- $\kappa \mathrm{B}$, which in turn induces inflammatory factors, such as IL-1 $\beta$, IL-6, IL-8, TNF- $\alpha$, monocyte chemotactic protein-1 (MCP-1), cyclooxygenase-2 (COX-2), and ICAM. ${ }^{72,73}$ ICAM-1 increases adhesion of monocytes and induces activation of protein kinase $\mathrm{C}(\mathrm{PKC})$, and down-regulates the expression of the zonulin (Zo) gene, contributing to retinal endothelial dysfunction. $^{74}$ Recent studies have shown that NLRP3 is activated in the retina of diabetic mice and human vitreous samples mediating retinal microvascular endothelial cells damage stimulated by hyperglycemia. ${ }^{75-77}$ The activation of NLRP3 inflammasome may be related to the pathogenesis of retinopathy. The expression levels of NLRP3 are upregulated in human retinal vascular endothelial cells (HRCECs) cultured with hyperglycemia and promote apoptosis of HRCECs. The apoptosis of HRCECs is decreased significantly when NLRP3 is silenced, and the NLRP3 inflammasome regulates the structure and function of BRB to induce damage of the retina. ${ }^{78}$

\section{Anti-Diabetes Drug Use}

Increased understanding of the interaction between use of anti-diabetes drugs and the microbiome could shed light on a correlation between intestinal flora and the occurrence and development of DR. Metformin is one of the most commonly used medications for the treatment of T2DM and its complications. Recently, a retrospective chart review study has shown that use of metformin is associated with a lower incidence of retinopathy in patients with T2DM. ${ }^{79}$ Metformin has both anti-angiogenic and anti-inflammatory effects on HRCECs by regulating NF- $\kappa \mathrm{B}$ p65, ICAM-1, MCP-1, and IL-8, and can reduce retinal neovascularization in Vldlr (-/-) mice and suppress leukocytosis in streptozotocin (STZ) induced diabetic mice. ${ }^{80}$ Additionally, the strong impact of metformin on the gut microbiome mediating its anti-diabetes effects was proposed. $^{81}$ Sodium glucose co-transporter 2 (SGLT2) inhibitors are widely applied in patients with diabetes to improve blood glucose level and prevent diabetes-related complications. Recently, Herat et al determined the protective effects of SGLT inhibitor alterations of gut microbiome; SGLT inhibition could decrease metabolic 
succinate level, increase SCFA butyric acid level and reduce norepinephrine content in diabetic mouse models. ${ }^{82}$ Succinate is an intermediate metabolite of gut metabolism which can be related with high incidence of DR. Succinate interacting with its specific receptor GPR91 can activate VEGF in retinal ganglion cells, thus aggravating the pathogenesis of DR. ${ }^{83}$ Taken together, there may be some potential changes in anti-diabetes treatment response and beneficial effects of metformin use against retinopathy, which will be related to different effects on the gut microbiome.

\section{Microbial Metabolism}

Microbial metabolites, including SCFA, glucogon-likepeptide-1 (GLP-1) and kynurenine (KYN), were proved to be associated with the development of DR. SCFA is a metabolic product of intestinal microbiome that breaks down carbohydrates or amino acids, such as acetic acid, propionate and butyrate. ${ }^{84}$ Hyperglycemia might result in disruption of the intestinal barrier and dysfunction of intestinal epithelial cells, and SCFAs absorption and utilization were the critical source of energy. ${ }^{85}$ In addition, it is worth noting that patients with T2DM commonly have a moderate degree of gut microbial dysbiosis, and the abundance of butyrate-producing bacteria is reduced, affecting the levels of SCFA. ${ }^{86,87}$ SCFA produced by colon fermentation binding to G-protein-coupled receptor 43 (GPR43) could regulate inflammation and GLP-1 secretion. Lixisenatide, a novel GLP-1 analog, has been confirmed to have vascular protective effects in diabetic rats. ${ }^{88}$ Liraglutide, which was approved by the FDA, in the application of T2DM was found to be associated with the reduction of gut microbial alpha diversity and the alteration of community distribution and the microbial interaction network. ${ }^{89}$ Meanwhile, another GLP-1 analog, exendin-4 (Ex-4), can reduce BRB permeability, and modulate the inflammatory response to LPS as well as inhibit NF- $\kappa$ B activation. ${ }^{90}$ Recently, it has been shown that GLP1 intervention reduces the levels of NADPH oxidase 3 (NOX3), superoxide dismutase 2 (SOD2), caspase 3 and light chain 3B (LC3B), while it activates the expression of B-cell lymphoma 2 (Bcl-2) in retina of type 2 diabetic rats, which suggests that GLP-1 treatment can alleviate apoptosis and autophagy of retinal cells. ${ }^{91}$ In particular, indoleamine 2, 3-dioxygenase (IDO) is thought to be responsible for tryptophan metabolism and promotes tryptophan to produce KYN. Tryptophan also produces derivatives such as indole-3-acetic acid (IAA) by intestinal bacterial metabolism. KYN is produced by irreversible transamination of kynurenine aminotransferase I (KAT-I) and kynurenine aminotransferase II (KAT-II). Further, KYNA is involved in retinal senescence and neurodegeneration. Studies have confirmed that there is a significant correlation between serum levels of IDO, KYN, and KYNA and the severity of DR in patients with diabetes. ${ }^{92}$

\section{Microbiota-Gut-Retina Axis}

Accumulating evidence reveals a reliable connection between gut microbiome and retina disease, which is considered to be a "microbiota-gut-retina axis" and plays a causal role in the onset and development of ocular diseases, including uveitis, glaucoma, AMD and DR. ${ }^{93,94}$ The axis proposed in recent studies demonstrates that the composition and disturbance of microbiome might potentially regulate body metabolism and serve as a source of inflammation and human systematic disease. ${ }^{95,96}$ Andriessen et al demonstrated that high-fat diet-induced obesity caused gut dysbiosis which can drive retinal inflammation and pathological angiogenesis in mouse models of laser photocoagulationinduced choroidal neovascularization (CNV). ${ }^{97}$ Rowan et al indicated that compositional and functional alterations in gut microbiota might influence the products of the microbiome; for instance, serotonin and carbohydrate nutrition are preventive against AMD using dietary glycemic-induced mouse models. ${ }^{98}$ Additionally, a series of observational researches has proven the potential role of micronutrient supplementation in the pathophysiology and rapid progression of ocular disease. ${ }^{99-102}$ Moreover, a better understanding of the basic mechanism of the microbiota-gut-retina axis can help investigators promote a new model of retinal disease care based on healthy eating habits and micronutrient intake. These practices can regulate the intestinal flora to reduce malnutrition, intestinal leakage and low glycemic index (LGI), and have protective abilities against retinal damage. ${ }^{103}$ Further research is needed to clarify whether the regulation of intestinal flora through dietary intervention can clinically delay the pathogenesis of retinal diseases.

\section{Conclusion and Outlook}

In short, the gut microbiota has been naturally known as a new human organ system that co-evolves with the host. Growing evidence suggests that gut microbiota plays a crucial role in the development of T2DM. Our review 
provided comprehensive evidence for the potential role of gut microbiota in the development of retinopathy. This review consists of the main mechanisms of DR, including hyperglycemia and inflammation, and alternative mechanisms which contain evidence that gut microbiota may be associated with DR. The hypothesis is that gut microbiota cause DR, microbial metabolism, and the microbiota-gut-retina axis. However, direct evidence of dysbiosis in DR are weak and further studies on cellular and molecular mechanisms and well-controlled human studies are needed for verification. Dissecting the gut microbiota through a comprehensive approach such as multiomics will help us understand the underlying pathogenic mechanisms, especially by focusing on the identification and functional analysis of specific regulatory species. These will provide essential information to modulate the potential role of gut microbiota in the prediction of retinopathy risk and therapeutic monitoring and are expected to decrease the onset and progression of retinopathy and prevent visual impairments in patients with diabetes.

\section{Acknowledgments}

We would like to thank Ms. Nan Jiang for English copywriting and language polishing and thank Dr. Cong Li for manuscript preparation.

\section{Funding}

This work was supported by the Science and Technology Program of Guangzhou, China (No. 202002020049), Project of Special Research on Cardiovascular Diseases (No. 2020XXG007), National Natural Science Foundation of China (No. 81300783; 82003882), China Postdoctoral Science Foundation (No. 2019TQ0358; 2019M661162), Liaoning Revitalization Talents Program (No. XLYC1807082), Shenyang Young and Middle-aged Science and Technology Innovation Talent Support Program (grant number RC190146). The funders had no role in the design and conduct of the study; the collection, management, analysis, interpretation of the data; the preparation, review, or approval of the manuscript; or the decision to submit the manuscript for publication.

\section{Disclosure}

The authors have declared that no competing interests exist.

\section{References}

1. Zhou H, Peng C, Huang D-S, Liu L, Guan P. microRNA expression profiling based on microarray approach in human diabetic retinopathy: a systematic review and meta-analysis. DNA Cell Biol. 2020;39(3):441-450. doi:10.1089/dna.2019.4942

2. Cecilia O-M, José Alberto C-G, José N-P, et al. Oxidative stress as the main target in diabetic retinopathy pathophysiology. J Diabetes Res. 2019;2019:1-21. doi:10.1155/2019/8562408

3. Sharma A. Emerging simplified retinal imaging. Dev Ophthalmol. 2017;60:56-62. doi:10.1159/000459690

4. Wong TY, Cheung CM, Larsen M, Sharma S, Simó R. Diabetic retinopathy. Nat Rev Dis Primers. 2016;2:16012. doi:10.1038/ nrdp. 2016.12

5. Gerstein HC, Werstuck GH. Dysglycaemia, vasculopenia, and the chronic consequences of diabetes. Lancet Diabetes Endocrinol. 2013;1(1):71-78. doi:10.1016/s2213-8587(13)70025-1

6. Antonetti DA, Klein R, Gardner TW. Diabetic retinopathy. $N$ Engl $J$ Med. 2012;366(13):1227-1239. doi:10.1056/NEJMra1005073

7. Maniadakis N, Konstantakopoulou E. Cost effectiveness of treatments for diabetic retinopathy: a systematic literature review. Pharmacoeconomics. 2019;37(8):995-1010. doi:10.1007/ s40273-019-00800-w

8. Yau JW, Rogers SL, Kawasaki R, et al. Global prevalence and major risk factors of diabetic retinopathy. Diabetes Care. 2012;35 (3):556-564. doi:10.2337/dc11-1909

9. Zhang G, Chen H, Chen W, Zhang M. Prevalence and risk factors for diabetic retinopathy in China: a multi-hospital-based cross-sectional study. $\mathrm{Br} \quad J \quad$ Ophthalmol. 2017;101 (12):1591-1595. doi:10.1136/bjophthalmol-2017-310316

10. Scirocco A, Matarrese P, Carabotti M, Ascione B, Malorni W, Severi C. Cellular and molecular mechanisms of phenotypic switch in gastrointestinal smooth muscle. $J$ Cell Physiol. 2016;231(2):295-302. doi:10.1002/jcp.25105

11. Rizk P, Barker N. Gut stem cells in tissue renewal and disease: methods, markers, and myths. Wiley Interdiscip Rev Syst Biol Med. 2012;4(5):475-496. doi:10.1002/wsbm.1176

12. Salarolli RT, Alvarenga L, Cardozo L, et al. Can curcumin supplementation reduce plasma levels of gut-derived uremic toxins in hemodialysis patients? A pilot randomized, double-blind, controlled study. Int Urol Nephrol. 2021;53:1231-1238. doi:10.1007/s11255-020-02760-z

13. Yang CY, Chen TW, Lu WL, et al. Synbiotics alleviate the gut indole load and dysbiosis in chronic kidney disease. Cells. 2021;10(1). doi:10.3390/cells10010114

14. Peng $\mathrm{J}, \mathrm{Lu} \mathrm{X}, \mathrm{Xie} \mathrm{K}$, et al. Dynamic alterations in the gut microbiota of collagen-induced arthritis rats following the prolonged administration of total glucosides of paeony. Front Cell Infect Microbiol. 2019;9:204. doi:10.3389/fcimb.2019.00204

15. Schoeler M, Caesar R. Dietary lipids, gut microbiota and lipid metabolism. Rev Endocr Metab Disord. 2019;20(4):461-472. doi:10.1007/s11154-019-09512-0

16. Verma $\mathrm{A}, \mathrm{Xu} \mathrm{K}, \mathrm{Du} \mathrm{T}$, et al. Expression of human ACE2 in lactobacillus and beneficial effects in diabetic retinopathy in mice. Mol Ther Methods Clin Dev. 2019;14:161-170. doi:10.1016/j.omtm.2019.06.007

17. Nadeem U, Xie B, Movahedan A, et al. High throughput RNA sequencing of mice retina reveals metabolic pathways involved in the gut-retina axis. bioRxiv. 2020. doi:10.1101/2020.10.01.318949

18. Huang Y, Wang Z, Ma H, et al. Dysbiosis and implication of the gut microbiota in diabetic retinopathy. Front Cell Infect Microbiol. 2021;11:646348. doi:10.3389/fcimb.2021.646348

19. Cheung N, Mitchell P, Wong TY. Diabetic retinopathy. Lancet (London, England). 2010;376(9735):124-136. doi:10.1016/ s0140-6736(09)62124-3 
20. Aiello LP. Diabetic retinopathy and other ocular findings in the diabetes control and complications trial/epidemiology of diabetes interventions and complications study. Diabetes Care. 2014;37 (1):17-23. doi:10.2337/dc13-2251

21. Hayes AJ, Leal J, Gray AM, Holman RR, Clarke PM. UKPDS outcomes model 2: a new version of a model to simulate lifetime health outcomes of patients with type 2 diabetes mellitus using data from the 30 year United Kingdom Prospective Diabetes Study: UKPDS 82. Diabetologia. 2013;56(9):1925-1933. doi:10.1007/s00125-013-2940-y

22. Kowluru RA, Kowluru A, Veluthakal R, et al. TIAM1-RAC1 signalling axis-mediated activation of NADPH oxidase-2 initiates mitochondrial damage in the development of diabetic retinopathy. Diabetologia. 2014;57(5):1047-1056. doi:10.1007/s00125-014-3194-z

23. Niimi N, Yako H, Takaku S, Chung SK, Sango K. Aldose reductase and the polyol pathway in schwann cells: old and new problems. Int J Mol Sci. 2021;22(3):1031. doi:10.3390/ ijms22031031

24. Kowluru RA, Mishra M. Oxidative stress, mitochondrial damage and diabetic retinopathy. Biochim Biophys Acta. 2015;1852 (11):2474-2483. doi:10.1016/j.bbadis.2015.08.001

25. Sahajpal N, Kowluru A, Kowluru RA. The regulatory role of Rac1, a small molecular weight GTPase, in the development of diabetic retinopathy. J Clin Med. 2019;8(7):965. doi:10.3390/jcm8070965

26. Mohammad G, Duraisamy AJ, Kowluru A, Kowluru RA. Functional regulation of an oxidative stress mediator, Rac1, in diabetic retinopathy. Mol Neurobiol. 2019;56(12):8643-8655. doi:10.1007/s12035-019-01696-5

27. Luo R, Xiao F, Wang P, Hu YX. lncRNA H19 sponging miR-93 to regulate inflammation in retinal epithelial cells under hyperglycemia via XBP1s. Inflamm Res. 2020;69(3):255-265. doi:10.1007/s00011-019-01312-1

28. Cvitkovic K, Sesar A, Sesar I, et al. Concentrations of selected cytokines and vascular endothelial growth factor in aqueous humor and serum of diabetic patients. Semin Ophthalmol. 2020;35(2):126-133. doi:10.1080/08820538.2020.1755320

29. Spilsbury K, Garrett KL, Shen WY, Constable IJ, Rakoczy PE. Overexpression of vascular endothelial growth factor (VEGF) in the retinal pigment epithelium leads to the development of choroidal neovascularization. Am J Pathol. 2000;157(1):135-144. doi:10.1016/s0002-9440(10)64525-7

30. Hachana S, Fontaine O, Sapieha P, Lesk M, Couture R, Vaucher E. The effects of anti-VEGF and kinin $\mathrm{B}(1)$ receptor blockade on retinal inflammation in laser-induced choroidal neovascularization. Br J Pharmacol. 2020;177(9):1949-1966. doi: $10.1111 /$ bph. 14962

31. Behar-Cohen F, Dernigoghossian M, Andrieu-Soler C, Levy R, Cohen R, Zhao M. Potential antiedematous effects of intravitreous anti-VEGF, unrelated to VEGF neutralization. Drug Discov Today. 2019;24(8):1436-1439. doi:10.1016/j.drudis.2019.05.034

32. Virgili G, Parravano M, Evans JR, Gordon I, Lucenteforte E. Anti-vascular endothelial growth factor for diabetic macular oedema: a network meta-analysis. Cochrane Database Syst Rev. 2018;10(10):Cd007419. doi:10.1002/14651858.CD007419.pub6

33. Ouyang H, Mei X, Zhang T, Lu B, Ji L. Ursodeoxycholic acid ameliorates diabetic retinopathy via reducing retinal inflammation and reversing the breakdown of blood-retinal barrier. Eur J Pharmacol. 2018;840:20-27. doi:10.1016/j.ejphar.2018.09.027

34. Gu R, Ding X, Tang W, Lei B, Jiang C, Xu G. A synthesized glucocorticoid- induced leucine zipper peptide inhibits retinal Müller cell gliosis. Front Pharmacol. 2018;9:331. doi:10.3389/ fphar.2018.00331

35. Ogura S, Kurata K, Hattori Y, et al. Sustained inflammation after pericyte depletion induces irreversible blood-retina barrier breakdown. JCI Insight. 2017;2(3):e90905. doi:10.1172/jci. insight.90905
36. Mitamura Y, Harada T, Harada C, et al. NF-kappaB in epiretinal membranes after human diabetic retinopathy. Diabetologia. 2003;46(5):699-703. doi:10.1007/s00125-003-1084-x

37. Li J, Zhao F, Wang Y, et al. Gut microbiota dysbiosis contributes to the development of hypertension. Microbiome. 2017;5(1):14. doi:10.1186/s40168-016-0222-x

38. Liu R, Hong J, Xu X, et al. Gut microbiome and serum metabolome alterations in obesity and after weight-loss intervention. Nat Med. 2017;23(7):859-868. doi:10.1038/nm.4358

39. Yu Y, Mao G, Wang J, et al. Gut dysbiosis is associated with the reduced exercise capacity of elderly patients with hypertension. Hypertens Res. 2018;41(12):1036-1044. doi:10.1038/s41440018-0110-9

40. Larsen N, Vogensen FK, van den Berg FW, et al. Gut microbiota in human adults with type 2 diabetes differs from non-diabetic adults. PLoS One. 2010;5(2):e9085. doi:10.1371/journal. pone.0009085

41. de Groot P, Nikolic T, Pellegrini S, et al. Faecal microbiota transplantation halts progression of human new-onset type 1 diabetes in a randomised controlled trial. Gut. 2021;70 (1):92-105. doi:10.1136/gutjnl-2020-322630

42. Khangwal I, Shukla P. Combinatory biotechnological intervention for gut microbiota. Appl Microbiol Biotechnol. 2019;103 (9):3615-3625. doi:10.1007/s00253-019-09727-w

43. Beli E, Yan Y, Moldovan L, et al. Restructuring of the gut microbiome by intermittent fasting prevents retinopathy and prolongs survival in db/db mice. Diabetes. 2018;67(9):1867-1879. doi: $10.2337 / \mathrm{db} 18-0158$

44. Gronbeck KR, Rodrigues CM, Mahmoudi J, et al. Application of tauroursodeoxycholic acid for treatment of neurological and non-neurological diseases: is there a potential for treating traumatic brain injury? Neurocrit Care. 2016;25(1):153-166. doi: 10.1007/s12028-015-0225-7

45. Rizzo G, Passeri D, De Franco F, et al. Functional characterization of the semisynthetic bile acid derivative INT-767, a dual farnesoid X receptor and TGR5 agonist. Mol Pharmacol. 2010;78(4):617-630. doi:10.1124/mol.110.064501

46. Wang XX, Wang D, Luo Y, et al. FXR/TGR5 dual agonist prevents progression of nephropathy in diabetes and obesity. JASN. 2018;29(1):118-137. doi:10.1681/asn.2017020222

47. Das T, Jayasudha R, Chakravarthy S, et al. Alterations in the gut bacterial microbiome in people with type 2 diabetes mellitus and diabetic retinopathy. Sci Rep. 2021;11(1):2738. doi:10.1038/ s41598-021-82538-0

48. Zhu L, Sha L, Li K, et al. Dietary flaxseed oil rich in omega-3 suppresses severity of type 2 diabetes mellitus via anti-inflammation and modulating gut microbiota in rats. Lipids Health Dis. 2020;19(1):20. doi:10.1186/s12944-0191167-4

49. Zaman GS, Zaman F. Relationship between postprandial endotoxemia in nonobese postmenopausal women and diabetic nonobese postmenopausal women. J Nat Sci Biol Med. 2015;6(1):89-93. doi:10.4103/0976-9668.149098

50. Layoun A, Santos MM. Bacterial cell wall constituents induce hepcidin expression in macrophages through MyD88 signaling. Inflammation. 2012;35(4):1500-1506. doi:10.1007/s10753-0129463-4

51. Kuwabara WMT, Yokota CNF, Curi R, Alba-Loureiro TC. Obesity and Type 2 Diabetes mellitus induce lipopolysaccharide tolerance in rat neutrophils. Sci Rep. 2018;8(1):17534. doi:10.1038/s41598-018-35809-2

52. Zhang L, Qin Q, Liu M, Zhang X, He F, Wang G. Akkermansia muciniphila can reduce the damage of gluco/lipotoxicity, oxidative stress and inflammation, and normalize intestine microbiota in streptozotocin-induced diabetic rats. Pathog Dis. 2018;76(4). doi:10.1093/femspd/fty028 
53. Chelakkot C, Choi Y, Kim DK, et al. Akkermansia muciniphila-derived extracellular vesicles influence gut permeability through the regulation of tight junctions. Exp Mol Med. 2018;50(2):e450. doi:10.1038/emm.2017.282

54. Campo GM, Avenoso A, Campo S, D'Ascola A, Nastasi G, Calatroni A. Molecular size hyaluronan differently modulates toll-like receptor-4 in LPS-induced inflammation in mouse chondrocytes. Biochimie. 2010;92(2):204-215. doi:10.1016/j. biochi.2009.10.006

55. Egshatyan L, Kashtanova D, Popenko A, et al. Gut microbiota and diet in patients with different glucose tolerance. Endocr Connect. 2016;5(1):1-9. doi:10.1530/ec-15-0094

56. Sohail MU, Althani A, Anwar H, Rizzi R, Marei HE. Role of the gastrointestinal tract microbiome in the pathophysiology of diabetes mellitus. J Diabetes Res. 2017;2017:9631435. doi:10.1155/ 2017/9631435

57. Sayed KM, Mahmoud AA. Heat shock protein-70 and hypoxia inducible factor- $1 \alpha$ in type 2 diabetes mellitus patients complicated with retinopathy. Acta Ophthalmol. 2016;94(5):e361-366. doi:10.1111/aos.12919

58. Abdulaal M, Haddad NM, Sun JK, Silva PS. The role of plasma kallikrein-kinin pathway in the development of diabetic retinopathy: pathophysiology and therapeutic approaches. Semin Ophthalmol. 2016;31(1-2):19-24. doi:10.3109/08820538.2015.1114829

59. Kim EJ, Lin WV, Rodriguez SM, Chen A, Loya A, Weng CY. Treatment of diabetic macular edema. Curr Diab Rep. 2019;19 (9):68. doi:10.1007/s11892-019-1188-4

60. Moubayed NM, Bhat RS, Al Farraj D, Dihani NA, El Ansary A, Fahmy RM. Screening and identification of gut anaerobes (Bacteroidetes) from human diabetic stool samples with and without retinopathy in comparison to control subjects. Microb Pathog. 2019;129:88-92. doi:10.1016/j.micpath.2019.01.025

61. Vagaja NN, Binz N, McLenachan S, Rakoczy EP, McMenamin PG. Influence of endotoxin-mediated retinal inflammation on phenotype of diabetic retinopathy in Ins2 Akita mice. Br J Ophthalmol. 2013;97(10):1343-1350. doi:10.1136/ bjophthalmol-2013-303201

62. Singh V, Yeoh BS, Vijay-Kumar M. Gut microbiome as a novel cardiovascular therapeutic target. Curr Opin Pharmacol. 2016;27:8-12. doi:10.1016/j.coph.2016.01.002

63. Karpova T, de Oliveira AA, Naas H, Priviero F, Nunes KP. Blockade of Toll-like receptor 4 (TLR4) reduces oxidative stress and restores phospho-ERK1/2 levels in Leydig cells exposed to high glucose. Life Sci. 2020;245. doi:10.1016/j.1fs.2020.117365

64. Rajamani U, Jialal I. Hyperglycemia induces Toll-like receptor-2 and -4 expression and activity in human microvascular retinal endothelial cells: implications for diabetic retinopathy. J Diabetes Res. 2014;2014:790902. doi:10.1155/2014/790902

65. Al-Obaide MAI, Singh R, Datta P, et al. Gut microbiota-dependent trimethylamine-N-oxide and serum biomarkers in patients with T2DM and advanced CKD. J Clin Med. 2017;6(9):86. doi:10.3390/jcm6090086

66. Nowiński A, Ufnal M. Trimethylamine N-oxide: a harmful, protective or diagnostic marker in lifestyle diseases? Nutrition (Burbank, Los Angeles County, Calif). 2018;46:7-12. doi:10.1016/j.nut.2017.08.001

67. Chen ML, Zhu XH, Ran L, Lang HD, Yi L, Mi MT. Trimethylamine-N-oxide induces vascular inflammation by activating the NLRP3 inflammasome through the SIRT3-SOD2mtROS signaling pathway. $J$ Am Heart Assoc. 2017;6(9). doi:10.1161/jaha.117.006347

68. Wang S, Du S, Lv Y, Wang W, Zhang F. Elevated microRNA-20b-3p and reduced thioredoxin-interacting protein ameliorate diabetic retinopathy progression by suppressing the NLRP3 inflammasomes. IUBMB Life. 2020;72:1433-1448. doi:10.1002/iub.2267
69. Wang $M$, Wang $Y$, Xie $T$, et al. Prostaglandin $E(2) / E P(2)$ receptor signalling pathway promotes diabetic retinopathy in a rat model of diabetes. Diabetologia. 2019;62(2):335-348. doi:10.1007/ s00125-018-4755-3

70. Wang P, Chen F, Wang W, Zhang XD. Hydrogen sulfide attenuates high glucose-induced human retinal pigment epithelial cell inflammation by inhibiting ROS formation and NLRP3 inflammasome activation. Mediators Inflamm. 2019;2019:8908960. doi:10.1155/2019/8908960

71. Chen H, Zhang X, Liao N, et al. Enhanced expression of NLRP3 inflammasome-related inflammation in diabetic retinopathy. Invest Ophthalmol Vis Sci. 2018;59(2):978-985. doi:10.1167/ iovs. 17-22816

72. Al-Ofi EA, Anumba DO. Ligands of toll-like receptors $2 / 4$ differentially alter markers of inflammation, adhesion and angiogenesis by monocytes from women with pre-eclampsia in co-culture with endothelial cells. $J$ Reprod Immunol. 2017;121:26-33. doi:10.1016/j.jri.2017.05.002

73. Wu M, Yang S, Wang S, et al. Effect of berberine on atherosclerosis and gut microbiota modulation and their correlation in high-fat diet-fed ApoE-/- mice. Front Pharmacol. 2020;11:223. doi:10.3389/fphar.2020.00223

74. Boini KM, Hussain T, Li PL, Koka S. Trimethylamine-N-oxide instigates NLRP3 inflammasome activation and endothelial dysfunction. Cell Physiol Biochem. 2017;44(1):152-162. doi:10.1159/000484623

75. Liu Q, Zhang F, Zhang X, et al. Fenofibrate ameliorates diabetic retinopathy by modulating Nrf2 signaling and NLRP3 inflammasome activation. Mol Cell Biochem. 2018;445(1-2):105-115. doi:10.1007/s11010-017-3256-X

76. Loukovaara S, Piippo N, Kinnunen K, Hytti M, Kaarniranta K, Kauppinen A. NLRP3 inflammasome activation is associated with proliferative diabetic retinopathy. Acta Ophthalmol. 2017;95(8):803-808. doi:10.1111/aos.13427

77. Wang Y, Tao J, Yao Y. Prostaglandin E2 activates NLRP3 inflammasome in endothelial cells to promote diabetic retinopathy. Hormone Metabol Res. 2018;50(9):704-710. doi:10.1055/a-0664-0699

78. Chen W, Zhao M, Zhao S, et al. Activation of the TXNIP/NLRP3 inflammasome pathway contributes to inflammation in diabetic retinopathy: a novel inhibitory effect of minocycline. Inflamm Res. 2017;66(2):157-166. doi:10.1007/s00011-016-1002-6

79. Li Y, Ryu C, Munie M, et al. Association of metformin treatment with reduced severity of diabetic retinopathy in type 2 diabetic patients. J Diabetes Res. 2018;2018:2801450. doi:10.1155/2018/ 2801450

80. Han J, Li Y, Liu X, et al. Metformin suppresses retinal angiogenesis and inflammation in vitro and in vivo. PLoS One. 2018;13(3): e0193031. doi:10.1371/journal.pone.0193031

81. Wu H, Esteve E, Tremaroli V, et al. Metformin alters the gut microbiome of individuals with treatment-naive type 2 diabetes, contributing to the therapeutic effects of the drug. Nat Med. 2017;23(7):850-858. doi:10.1038/nm.4345

82. Herat LY, Ward NC, Magno AL, et al. Sodium glucose co-transporter 2 inhibition reduces succinate levels in diabetic mice. World J Gastroenterol. 2020;26(23):3225-3235. doi:10.3748/wjg.v26.i23.3225

83. Sapieha P, Sirinyan M, Hamel D, et al. The succinate receptor GPR91 in neurons has a major role in retinal angiogenesis. Nat Med. 2008;14(10):1067-1076. doi:10.1038/nm.1873

84. Cummings JH, Pomare EW, Branch WJ, Naylor CP, Macfarlane GT. Short chain fatty acids in human large intestine, portal, hepatic and venous blood. Gut. 1987;28(10):1221-1227. doi:10.1136/gut.28.10.1221

85. Zhao L, Zhang F, Ding X, et al. Gut bacteria selectively promoted by dietary fibers alleviate type 2 diabetes. Science. 2018;359 (6380):1151-1156. doi:10.1126/science.aao5774 
86. Zhao L, Lou H, Peng Y, Chen S, Zhang Y, Li X. Comprehensive relationships between gut microbiome and faecal metabolome in individuals with type 2 diabetes and its complications. Endocrine. 2019;66(3):526-537. doi:10.1007/s12020-019-02103-8

87. Yamaguchi Y, Adachi K, Sugiyama T, et al. Association of intestinal microbiota with metabolic markers and dietary habits in patients with type 2 diabetes. Digestion. 2016;94(2):66-72. doi:10.1159/000447690

88. Abdel-Latif RG, Heeba GH, Taye A, Khalifa MMA. Lixisenatide, a novel GLP-1 analog, protects against cerebral ischemia/reperfusion injury in diabetic rats. Naunyn Schmiedebergs Arch Pharmacol. 2018;391(7):705-717. doi:10.1007/s00210-0181497-1

89. Shang J, Liu F, Zhang B, et al. Liraglutide-induced structural modulation of the gut microbiota in patients with type 2 diabetes mellitus. PeerJ. 2021;9:e11128. doi:10.7717/peerj.11128

90. Gonçalves A, Lin CM, Muthusamy A, et al. Protective effect of a GLP-1 analog on ischemia-reperfusion induced blood-retinal barrier breakdown and inflammation. Invest Ophthalmol Vis Sci. 2016;57(6):2584-2592. doi:10.1167/iovs.15-19006

91. Cai X, Li J, Wang M, et al. GLP-1 treatment improves diabetic retinopathy by alleviating autophagy through GLP-1R-ERK1/ 2-HDAC6 signaling pathway. Int $J$ Med Sci. 2017;14 (12):1203-1212. doi:10.7150/ijms.20962

92. Munipally PK, Agraharm SG, Valavala VK, Gundae S, Turlapati NR. Evaluation of indoleamine 2,3-dioxygenase expression and kynurenine pathway metabolites levels in serum samples of diabetic retinopathy patients. Arch Physiol Biochem. 2011;117 (5):254-258. doi:10.3109/13813455.2011.623705

93. Rinninella E, Mele MC, Merendino N, et al. The role of diet, micronutrients and the gut microbiota in age-related macular degeneration: new perspectives from the gutretina axis. Nutrients. 2018;10(11):1677. doi:10.3390/nu10111677

94. Chaiwiang N, Poyomtip T. Microbial dysbiosis and microbiota-gut-retina axis: the lesson from brain neurodegenerative diseases to primary open-angle glaucoma pathogenesis of autoimmunity. Acta Microbiol Immunol Hung. 2019;66 (4):541-558. doi:10.1556/030.66.2019.038
95. Turnbaugh PJ, Hamady M, Yatsunenko T, et al. A core gut microbiome in obese and lean twins. Nature. 2009;457 (7228):480-484. doi:10.1038/nature07540

96. Zinkernagel MS, Zysset-Burri DC, Keller I, et al. Association of the intestinal microbiome with the development of neovascular age-related macular degeneration. Sci Rep. 2017;7:40826. doi: $10.1038 /$ srep40826

97. Andriessen EM, Wilson AM, Mawambo G, et al. Gut microbiota influences pathological angiogenesis in obesity-driven choroidal neovascularization. EMBO Mol Med. 2016;8(12):1366-1379. doi:10.15252/emmm.201606531

98. Rowan S, Jiang S, Korem T, et al. Involvement of a gut-retina axis in protection against dietary glycemia-induced age-related macular degeneration. Proc Natl Acad Sci U S A. 2017;114(22): E4472-e4481. doi:10.1073/pnas.1702302114

99. Hammond BR, Fletcher LM, Roos F, Wittwer J, Schalch W. A double-blind, placebo-controlled study on the effects of lutein and zeaxanthin on photostress recovery, glare disability, and chromatic contrast. Invest Ophthalmol Vis Sci. 2014;55 (12):8583-8589. doi:10.1167/iovs.14-15573

100. Braakhuis A, Raman R, Vaghefi E. The association between dietary intake of antioxidants and ocular disease. Diseases (Basel, Switzerland). 2017;5(1). doi:10.3390/diseases5010003

101. Souied EH, Delcourt C, Querques G, et al. Oral docosahexaenoic acid in the prevention of exudative age-related macular degeneration: the nutritional AMD treatment 2 study. Ophthalmology. 2013;120(8):1619-1631. doi:10.1016/j.ophtha.2013.01.005

102. Aoki A, Inoue M, Nguyen E, et al. Dietary n-3 fatty acid, $\alpha-$ tocopherol, zinc, vitamin $\mathrm{D}$, vitamin $\mathrm{C}$, and $\beta$-carotene are associated with age-related macular degeneration in Japan. Sci Rep. 2016;6:20723. doi:10.1038/srep20723

103. Zhang QY, Tie LJ, Wu SS, et al. Overweight, obesity, and risk of age-related macular degeneration. Invest Ophthalmol Vis Sci. 2016;57(3):1276-1283. doi:10.1167/iovs.15-18637
Journal of Inflammation Research

\section{Publish your work in this journal}

The Journal of Inflammation Research is an international, peerreviewed open-access journal that welcomes laboratory and clinical findings on the molecular basis, cell biology and pharmacology of inflammation including original research, reviews, symposium reports, hypothesis formation and commentaries on: acute/chronic inflammation; mediators of inflammation; cellular processes; molecular mechanisms; pharmacology and novel anti-inflammatory drugs; clinical conditions involving inflammation. The manuscript management system is completely online and includes a very quick and fair peerreview system. Visit http://www.dovepress.com/testimonials.php to read real quotes from published authors. 